\title{
Qualitative Analysis of Students' Epistemic Framing Surrounding Instructor's Interaction
}

\author{
Amogh Sirnoorkar, ${ }^{1}$ Christopher A. F. Hass, ${ }^{1}$ Qing X. Ryan, ${ }^{2}$ and Eleanor C. Sayre ${ }^{1}$ \\ ${ }^{1}$ Department of Physics, Kansas State University, Manhattan, KS 66506 \\ ${ }^{2}$ California State Polytechnic University, Pomona, CA 91768
}

As part of a larger study of exploring students' problem solving in small groups, we investigated how the instructor influences students' epistemic framing in an upper-division electromagnetism class. We use the CAMP (Conceptual, Algorithmic, Mathematics, Physics) framework to investigate the dynamics of students' frames surrounding their interaction with the instructor, tracking frame triplets before, during and after the instructor's intervention during tutorial sessions. We present a case study of one such triplet (AP, CP, AP) by qualitatively analyzing student interaction with the instructor. Careful examination indicates that disagreements during the interaction necessitated the instructor to initiate conceptual discussion thereby causing a frame shift from an algorithmic to a conceptual frame. Further distribution must maintain the cover page and attribution to the article's authors. 


\section{INTRODUCTION}

Students' epistemic framing during problem solving attracts considerable attention in physics education research [1-10]. While existing literature indicates that instructors can influence students' framing during collaborative problem solving [11], we explore the mechanisms by which this occurs. We investigate this influence qualitatively by employing the CAMP (Conceptual, Algorithmic, Mathematics and Physics) [4] framework and analyzing frame triplets. A frame triplet is set of three consecutive, discrete frames centered around the instructor's intervention. The first frame in a triplet is the students' starting frame, the second is the framing of the instructor's interaction and the last is the students' frame after the instructor's exit. Prior work [11] using the same data suggests that while students spent substantial time in algorithmic frames, the instructor prefers to respond conceptually. Building on this work, we investigate how interaction with the instructor may facilitate a transition of students' frames from an algorithmic to a conceptual frame. We select a triplet in which a conceptual frame is sandwiched between two algorithmic ones. We seek to understand the features of the instructor's interaction which led her to shift the frame from algorithmic to conceptual.

We present a case study drawn from an upper division electromagnetism (E\&M) tutorial. The students are engaged in the calculation of electric potential due to a finite line of charge along the axis of the charge distribution. When they reach a dead end the instructor intervenes. We find that instances of disagreements during the interaction prompt the instructor to provide additional conceptual input. This results in predominantly conceptual framing during the interaction. Disagreements during this interaction are an anchoring feature prompting the instructor to nudge students towards a conceptual discussion. Through this result, we hope to explore the verbal cues in students-teacher interaction which may help facilitate student learning in normal classroom settings.

FIG. 1: CAMP frames

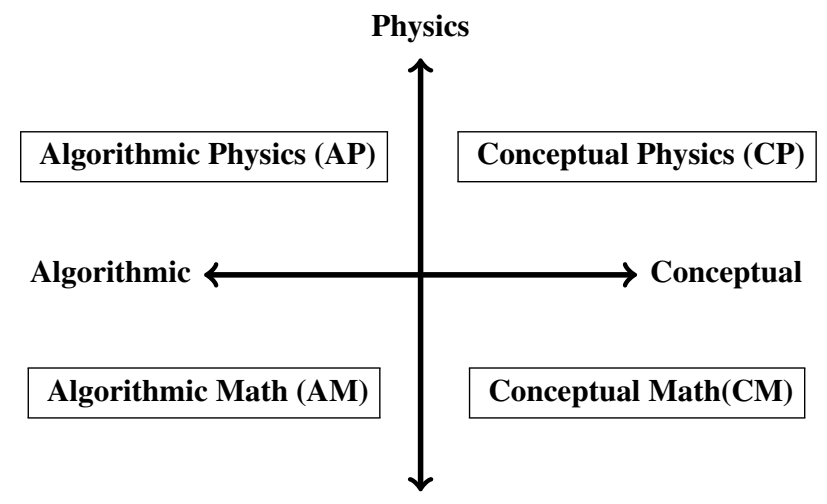

Mathematics
Disagreements can disturb the flow of a conversation when participants realize they hold contradictory beliefs. A disagreement is defined as an expression of a view that differs from that expressed by another speaker [12]; during a disagreement, common ground must be established before the discourse can proceed [13, 14]. This is often achieved through clarification, justification, or explanation. In collaborative problem solving involving an instructor, disagreements usually represent students' ideas being challenged or contradicted. Such instances often require deeper explanations from the instructor to address their concerns. Disagreements during a physics discourse can affect students' understanding of the context. They are instrumental in managing discussions amongst students for collaborative scientific sensemaking and critically evaluating each others' viewpoints [15].

\section{THEORETICAL FRAMEWORK}

We employ the two dimensional CAMP framework $[4,5$, $11]$ to investigate students' epistemic frames. This framework associates students' activities during problem solving along two mutually orthogonal axes yielding four epistemic frames as shown in Figure 1. Productive problem solving requires appropriate frames as well as effective transitions (shifts) between them. Earlier work has analyzed students' frame shifts in the absence of the instructor's assistance [4, 5], and between students and instructors [11]. In this work, we extend prior work on students' frame shifting $[4,5]$ and instructors' influence on student framing [11] to extend analysis of frame triplets. A triplet (explained in the previous section) with similar frames could involve both students and the instructor following each other's leads, as is common in this data set [11]. On the other hand, dissimilar frames may imply that students are stuck in a frame and the instructor responds with new information from another frame. This intervention from the instructor may result in students either continuing to work with their initial framing or make the necessary transition to a new one. As conceptual physics seems to be the instructor's preferred frame [11], it would be interesting to observe a triplet with dissimilar frames wherein the instructor's interaction is marked with conceptual physics.

The instructor's assistance during problem solving often involves upholding students' existing ideas or introducing new ones with incidental verbal disagreements. Furthermore, these disagreements can be either from the instructor or student end. Under classroom settings, teachers occasionally attend to disagreements by invoking their authority through their expertise $[16,17]$. In this process of resolving disagreements we believe that students' ideas on the content are negotiated. In the present work, we observe two types of disagreements between students and the instructor. The first in which there is an explicit use of negating language (e.g. "no, that's not right") called 'Bald on record' and the other involving indirect mitigation (e.g. through questions) $[14,18]$. 


\section{METHODOLOGY}

The video data in this study is of a tutorial session from the initial stages of an undergraduate upper-division E\&M course from 2015 at a large midwestern university. The students are engaged in tutorial problems [19] with a common shared whiteboard between them. The instructor circulates, interacting with each group of 3-5 students regularly. We focus on a group of three male students; the instructor is female.

We selected interactions based on content richness and their audio and visual clarity. Prior work on the same data indicates students spend substantial time in algorithmic frames and while the instructor prefers a conceptual frame. We identify and focus on the conceptual nature of the instructor's interaction in a triplet with dissimilar frames - AP, CP, AP. This intervention includes the entire duration of the instructor's presence at the table (90 seconds). To code the frames before and after the intervention, we examined one minute before and after, total time 210 seconds $(60+90+60)$.

Students' words, prosody, and body gestures were used by the first three authors to apply a previously developed coding scheme [4], using a minimum of 30 seconds of actions to determine a frame. After discussing coding discrepancies, consensus was reached on the coding and on the description of the chosen scenarios.

We focus on the 'turning points' in the conversation which indicated the interaction's framing to be predominantly conceptual. We present this case and its analysis in the following sections. The scenario involves a group of three students ( $\mathrm{S} 1$, $\mathrm{S} 2$, and S3) trying to determine the expression for electric potential at a point along the $z$-axis due to a line of charge placed between $z=0$ and $z=-d$ as shown in Fig. 2 (a).

\section{ANALYSIS}

\section{A. Before interaction}

The students begin by writing the potential integral in terms of volume charge density with an equation taken from the problem statement. To deal with the charge density term, they set up another integral $Q=\int_{0}^{d} \lambda d l$, which upon evaluation yields $Q=\lambda d$, a constant (see Fig. 2 (b) and (c)). They then set out to determine the expression for the potential. They begin by exploring the meaning of $\boldsymbol{r}$ in general (which is the distance between the charge element $d q$ and the point of interest $P$ ) and then in the given context with the help of Cartesian coordinate system. The meaning of $\boldsymbol{r}$ reaffirms their obtained line integral. This integral on further evaluation yields an expression in terms of $\ln (0)$ and $\ln (\infty)$ as shown in FIG. 2 (b). This is when students realize that something has gone wrong and hit a dead end. At this stage the instructor arrives, observes the students being stuck and intervenes.

Before the instructor's arrival, the students engage in setting up the required equations and meticulous handling of the integral. We code this behavior as algorithmic physics (AP). We observe two major features of their work in this frame

1. Student $\mathrm{S} 1$ believes that the line of charge behaves like a point charge when viewed end on from any point along the $z$-axis. (Note that the line charge does look like a point in that perspective, but the potential due to this line charge is not the same as that of a point charge).

2. Students set the limits of the integral to be limits used in calculating potential difference (from 0 to $\infty$ ) instead of the locations of the charge (from $-d$ to 0 ). This is congruent to prior findings about students' difficulties in E\&M [20].

The students do not explicitly discuss observation (1) among themselves until S1 mentions this in the presence of the instructor. However, S1 explicitly advocates for the observation (2) and all group members unanimously agreed.

\section{B. During interaction: Episode I}

Arriving at the table, the instructor observes the students working on the whiteboard for 15s. She then initiates the conversation with: "Yeah, there are some issues here". Pointing at the written integral (the equation given in the problem statement) she suggests "This is definitely true, but I would like to suggest that instead of doing volume integral, you could just do a line integral". She continues "like you were doing here" by pointing towards students' equation: $Q=\int_{0}^{d} \lambda d l$. While this is being said, S1 erases the whiteboard to make space for the instructor as the others listen.

The initial comment from the instructor ("This is definitely true, but...") might mirror a sense of disagreement. But, this is not in response to students' verbal statement, but rather a reflection of their engagement with the problem and their writing on the whiteboard. Further, the structure of her remarks ( “... but... instead of. . you could") indicates her noticing the students' resolution of the dissimilarity between two integrals: the volume integral (from the problem statement) and the line integral (written by students). And lastly, her other statement "like you were doing here" reflects her efforts in understanding the students' written work.

At this point, $\mathrm{S} 1$ asks the Instructor (I):

S1: "Well, this is. . since we are looking along the line on the z-axis, then this line just looks like a point charge?"

I: "No, it does not... does not look like only a point charge". (Pointing towards the figure illustrating the linear charge) "So these pieces contribute less than these pieces".

Looking at S1's inquiry, we infer that students' work can be explained by their idea of viewing the entire line of charge as a point where they can just treat the total charge as $Q$. Instructor responds to this view by explaining that the charge elements which are farther away from point $P$ contribute less 


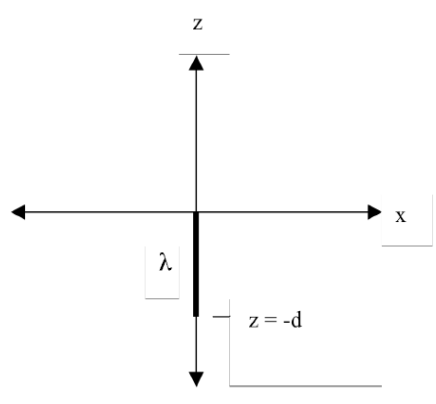

(a) Given figure in the problem

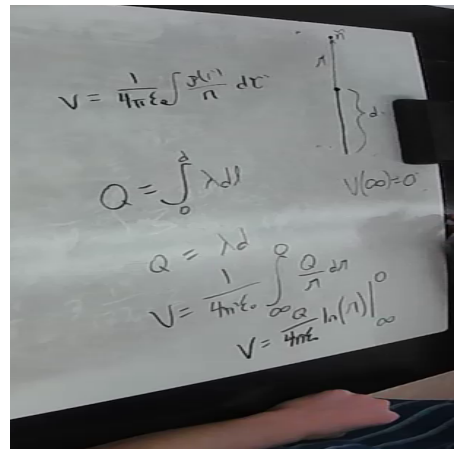

(b) Students' work

$$
\begin{aligned}
& V=\frac{1}{4 \pi \epsilon_{0}} \int \frac{\rho\left(z^{\prime}\right) d \tau^{\prime}}{d \tau} \\
& Q=\int \lambda d l \\
& Q=\lambda d \\
& V=\frac{1}{4 \pi \epsilon_{0}} \int_{\infty}^{0} \frac{Q}{\tau} d \tau \\
& V=\left.\frac{Q}{4 \pi \epsilon_{0}} \ln \tau\right|_{\infty} ^{0}
\end{aligned}
$$

(c) Whiteboard transcript

FIG. 2: Problem statement: A uniform linear charge density $\lambda$ extends from the origin to the point $(0,0,-d)$. Using the $r$ technique from earlier in the course, find an expression for the potential for points on the positive z-axis, $V(z)$. Remember:

$$
V=\frac{1}{4 \pi \epsilon_{0}} \int \frac{\rho\left(\boldsymbol{z}^{\prime}\right) d \tau^{\prime}}{d \boldsymbol{r}}
$$

to the potential than those which are nearer. Students listen keenly to the explanatory tone of the instructor until she makes her next move.

This episode assumes significance since this is the first instance of a disagreement between students and the instructor emerging in this conversation. The statement of the instructor "No, it does not..." clearly falls under the category of a negating linguistic indicator thus making the disagreement to be 'Bald on record' ( [14]) type. Secondly, the instructor did not stop after disagreeing but rather went ahead with a supplementary conceptual explanation on the 'why' of her response.

\section{During interaction: Episode II}

Moving forward, the instructor makes modifications to the students' integral on the whiteboard to make it relevant to the given context: reducing the equation from volume to a line integral and replacing volume charge density $(\rho)$ with linear charge density $(\lambda)$. During one such alteration, S1 refers to the limits and says:

S1: "infinity to zero"

I: "Where is the charge? Infinity to zero?"

S1: "The charge or the potential?"

I: "The charge, (while pointing at potential integral) because you are doing this integral where the charge is"

The question from the instructor "Where is the charge? Infinity to zero?" contradicts the assumption which the students had been working under (2). As mentioned earlier, students agreed that the limits of the integral were determined by the potential rather than by the charge distribution. In this context, we resonate with Janie Rees-Miller's view on classroom disagreements that it is sufficient for an utterance to be a disagreement (indirect) even if not stated explicitly and students' questions are often indirect and can be 'cloaked in the guise of an information question' [18]. We interpret S1's question as indirect disagreement. This disagreement prompted the instructor to further explain why location of charge distribution matters in determining the limits of the integral. Meanwhile S3 makes an intervening comment on the limits "zero to negative d" to which she agrees by replying "zero to negative $d$, then those are the limits of your integral".

\section{During interaction: Episode III}

Near the end of the interaction, the instructor points out modifications that need to be made in the integral, this time turning to $d r$. She suggests, " $d r$ depends on which little piece of charge you are looking at. So, you wanna plug in $d r$ (while erasing the $d \tau)$ to be the distance from your little piece of charge to the point of interest. That distance will vary based on where you are looking at."

She then poses a probing question "So what is $d z$ ?". S3 replies while gesturing his hand in upward direction "infinitely far away". The instructor in turn disagrees with the student: "not infinitely far away" bringing out our third and the last disagreement of this interaction. She chooses to follow up her disagreement by reiterating the meaning of $d z$ as "It's from your piece of charge dq to your point of interest". Similar to the arguments made in Episode IVB, we believe the disagreeing statement "not infinitely far away" to be of the 'Bald on record' type with explicit use of negating ('not') linguistic indicator. This disagreement engendered a discussion on the contextual meaning of $d \tau$.

Continuing, the instructor gestures on the diagram, and suggests "So, I am gonna suggest that you write dr as sum of zero (from origin) to your place of charge and zero to your place of interest". With this comment she conveys $d \boldsymbol{r}$ to be the distance from the position of $d q$ (which varies) to point $\mathrm{P}$ (which is constant). 
Because all three of these episodes of the students interacting with the instructor were dominated by conceptual considerations - reasoning of charge elements contributing unevenly to the potential, the limits of the integral being determined by the charge distribution and elucidating the contextual meaning of $d r$ - we interpret this as an extended period of being in the conceptual physics (CP) frame.

\section{E. After interaction}

After the instructor's exit from the table, the students resume their work by picking up the clues from the interaction. However, we find no signs of frustration or lack of enthusiasm in students due to the apparent setbacks from the instructor's intervention. Following the suggestion of the instructor just before her exit, students revisit the meaning of $\boldsymbol{r}$ by writing it in vector form as $\overrightarrow{\boldsymbol{r}}=(\vec{r}-\vec{d})$ and then plugging it in the revised potential integral. These set of activities mark the post-60 seconds interval of instructor's intervention. Since the students are mainly exploring known physics equations and rearranging them, we code this duration (the last frame of the triplet) to be algorithmic physics (AP).

This is the final frame shift of the triplet, from conceptual physics during the instructor's interaction to algorithmic physics (AP) after the interaction. However, this final shift does not appear to be triggered by disagreements, but rather corresponds to resuming the natural flow of problem solving after resolving their initial issues (observations 1 and 2).

\section{DISCUSSION}

Across the instructor interaction, we observe three instances of disagreements between the instructor and students. The first instance of disagreement on the linear charge distribution acting as a point charge, the second on the limits of the potential integral and the last pertaining to the contextual meaning of $d r$. These instances were instrumental in prompting the instructor to initiate a supplementary discussion. Before and after the intervention, the students are in the algorithmic physics (AP) frame; during it, they are in the conceptual physics (CP) frame.

The instructor at the beginning of the interaction picked up the existing students' idea (potential integral) and modified each term in accordance with the context of the problem. Because the students are in algorithmic physics before and after the instructor's intervention, it would not have been surprising had the middle frame in the triplet revolved around following procedures of formulating and evaluating the line integral. In that case, the central part of our triplet would have been algorithmic physics (AP). Instead, the instructor's interaction is built around the conceptual underpinnings of the problem.

One possible reason for the occurrence of a conceptual physics framing, when surrounded by algorithmic physics, is because a disagreement has occurred. Prompted by these three disagreements during the interaction, we observe a frame shift from algorithmic physics (AP) in students' initial frame to a sustained conceptual physics (CP) framing during the interaction.

\section{CONCLUSION}

Prior work indicates that both students and instructors can influence each other's epistemic framing during collaborative problem solving. To qualitatively investigate the mechanism of this influence, we take up the idea of a frame triplet and chose to focus on the instructor-led frame shift from algorithmic physics (AP) to conceptual physics (CP). In our case study, we identified disagreements during the interaction with the instructor as likely anchoring features responsible for the sustained conceptual nature of interaction's framing. This in turn resulted in the frame shift from an algorithmic to a conceptual frame.

While the instructor engaged in 'Bald on record' disagreement, the students used implicit ones. When those disagreements are resolved, the students return to an algorithmic frame (AP). Further, we believe disagreements to be one among many other features responsible for influencing students' framing during instructor's intervention. Other features like questions posed, context and purpose of the interaction, nature of the problem (involving mathematical computation vs conceptual reasoning) etc. may also contribute in promoting frame shifts. Further investigations by taking these features into account may shed better insight on the above mentioned influence.

Due to the exploratory nature of this study, we only analyzed one episode in detail and chose to focus on conceptual nature of instructor's interaction. However, conceptual physics need not be the 'preferred' frame during problem solving. We opine that the choice of each frame carries its own merit based on the context of the problem being solved. Thus, exploring the 'fluidity' of frame transitions involving other frames (conceptual and algorithmic math) can equally contribute in understanding the mechanism of instructor's influence. In addition, more analyses in the future similar to the present work can further explore within the domain of disagreements ('Bald on record' vs implicit) and how disagreements interact with conceptual or algorithmic framing. Our future work may also investigate pedagogical implications for how instructors could support students' problem solving and productive frame shifting.

\section{ACKNOWLEDGMENTS}

Thanks to Lydia Bender, Amali Priyanka Jambuge, Hien Khong and Maxwell Franklin for their valuable contributions. Portions of this work were supported by NSF DUE-1430967 and the KSU and CPP departments of physics. 
[1] R. E. Scherr and D. Hammer, Student behavior and epistemological framing: examples from collaborative active learning activities Physics, Cognit. Instr. 27, 147 (2009).

[2] P. W. Irving, M. S. Martinuk and E. C. Sayre, Transitions in students' epistemic framing along two axes, Phys. Rev. ST Phys. Educ. Res. 9, 010111 (2013).

[3] J. Tuminaro and E. F. Redish, Elements of a cognitive model of physics problem solving: Epistemic games, Phys. Rev. ST Phys. Educ. Res., 3, 020101, (2007).

[4] H. D. Nguyen, D. N. Chari, and E. C. Sayre, Dynamics of students' epistemological framing in group problem solving, Eur. J. Phys., 37, 065706 (2016).

[5] B. Modir, J. D. Thompson, and E. C. Sayre, Students' epistemological framing in quantum mechanics problem solving, Phys. Rev. Phys. Educ. Res., 13, 020108, (2017).

[6] T. J. Bing, and E. F. Redish, Analyzing problem solving using math in physics: Epistemological framing via warrants, Phys. Rev. ST Phys. Educ. Res., 5, 020108, (2009).

[7] A. Elby, and D. Hammer, Epistemological resources and framing: A cognitive framework for helping teachers interpret and respond to their studentsâ epistemologies, Personal epistemology in the classroom: Theory, research, and implications for practice, 409-434, (Cambridge University Press, 2010).

[8] P. Hutchison and D. Hammer, Attending to student epistemological framing in a science classroom. Sci. Educ., 94(3), 506524, (2010).

[9] D. Hammer, A. Elby, R. E. Scherr, and E. F. Redish, Resources, framing, and transfer. Transfer of learning from a modern multidisciplinary perspective, 89, (2005).

[10] M. E. Hing-Hickman, Epistemic strategies for solving two- dimensional physics problems, (2011)

[11] D. N. Chari, and H. D. Nguyen, and D.A. Zollman, and E. C. Sayre, Student and instructor framing in upper-division physics, Am. J. Phys., 87, 875, (2019).

[12] M. Sifianou, The use of diminutives in expressing politeness: Modern Greek versus English, J. Pragmat. 17, 155, (1992).

[13] K. Ehlich, and J. Wagner, The discourse of business negotiation, Vol. 8, (Walter de Gruyter, 2011).

[14] R. Lopez-Ozieblo, Disagreeing without a 'no': How teachers indicate disagreement in a Hong Kong classroom, J. Pragmat., 137, 1, (2018).

[15] L. D. Conlin and R.E. Scherr, Making Space to Sensemake: Epistemic Distancing in Small Group Physics Discussions, Cognition and Instruction, 396-423, (2018)

[16] B.K. Sharma, Enactment of teacher identity in resolving student disagreements in small group peer interactions, Linguistics and Education, 24, 247, (2013).

[17] N. Markee, Zones of interactional transition in ESL classes, Mod. Lang. J., 88, 853, (2004).

[18] J. Rees-Miller, Power, severity, and context in disagreement, J. Pragmat., 32, 1087, (2000).

[19] B. Charles , D. Michael, and J. P. Steven, Developing Tutorials for Advanced Physics Students : Processes and Lessons Learned, in 2013 Physics Education Research Conference Proceedings, p. 61-64.

[20] R.E. Pepper, and S.V. Chasteen, and S. J. Pollock, and K. K. Perkins, , Observations on student difficulties with mathematics in upper-division electricity and magnetism, Phys. Rev. ST Phys. Educ. Res., 8, 010111, (2012). 